\title{
Precision Lamb-shift polarimeter for polarized atomic and ion beams
}

\author{
R. Engels, ${ }^{\text {a) }}$ R. Emmerich, J. Ley, G. Tenckhoff, and H. Paetz gen. Schieck \\ Institut für Kernphysik der Universität zu Köln, 50937 Köln, Germany \\ M. Mikirtytchiants \\ Institut für Kernphysik, Forschungszentrum Jülich, 52425 Jülich, Germany \\ and St. Petersburg Nuclear Physics Institute, 188300 Gatchina, Russia
}

F. Rathmann and H. Seyfarth

Institut für Kernphysik, Forschungszentrum Jülich, 52425 Jülich, Germany

\section{A. Vassiliev \\ St. Petersburg Nuclear Physics Institute, 188300 Gatchina, Russia}

(Received 20 May 2003; accepted 18 August 2003)

\begin{abstract}
The Lamb-shift polarimeter described here enables the polarization of a beam of hydrogen (deuterium) atoms, or of a slow proton (deuteron) beam, to be measured with an absolute precision better than $1 \%$ within a few seconds. The polarimeter measures the intensity ratios of Lyman- $\alpha$ transitions after Stark quenching of metastable hyperfine substates that were selected in a spin filter. For that purpose the hydrogen (deuterium) atoms are ionized in a Glavish-type ionizer. By charge exchange of the protons (deuterons) in cesium vapor, atoms in the metastable $2 S$ state are produced. For a hydrogen beam of $3 \times 10^{16}$ atoms/s, $\sim 3 \times 10^{6}$ photons/s are registered in a photomultiplier, i.e., the polarimeter efficiency is about $10^{-10}$. The signal-to-background ratio in the Lyman- $\alpha$ spectrum is excellent, thus beam intensities of one to two orders of magnitude less would still be sufficient to carry out a precise measurement. The different components of the polarimeter affect the measured polarization in several ways. It was, therefore, crucial to determine precisely the associated correction factors in order to derive the nuclear polarization from the measured Lyman- $\alpha$ spectra. (C) 2003 American Institute of Physics. [DOI: 10.1063/1.1619550]
\end{abstract}

\section{INTRODUCTION}

A number of Lamb-shift polarimeters (LSPs) of different designs ${ }^{1-4}$ have been in use to measure the polarization of proton (deuteron) beams at polarized ion sources. Some others are being planned. ${ }^{5-7}$ The present LSP was designed, built, and tested with unpolarized ion beams at the Universität zu Köln. The measurements with polarized atomic beams have been carried out at the Forschungszentrum Jülich using the ANKE polarized atomic beam source (ABS). ${ }^{8}$ The LSP was specifically developed to measure and optimize the polarization of neutral beams from such beam sources with higher absolute accuracy in shorter times than achieved previously.

In the present article, it is shown that it is possible to measure the polarization of an atomic beam of hydrogen (deuterium) and also of a slow proton (deuteron) beam up to $2 \mathrm{keV}(4 \mathrm{keV})$ energy with a precision of $1 \%$ in a few seconds. Beam intensities of less than $10^{15}$ atoms/s or $10^{11}$ protons/s are required. Therefore, polarization measurements of the gas in a storage cell target appear feasible with a small number of atoms effusing from the cell. Thus, our LSP constitutes an alternative to polarimeters of the BreitRabi type. ${ }^{9}$

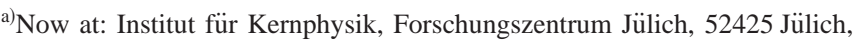
Germany; electronic mail: r.w.engels@fz-juelich.de
}

\section{EXPERIMENTAL SETUP}

The functions of the following LSP components are:

- An ionizer with a strong magnetic field ionizes the (polarized) atoms from an atomic beam.

- A Wien filter is used to provide a longitudinal polarization direction for the atoms. It also removes unwanted charged particles from the beam.

- A cesium vapor cell transforms the ions into metastable atoms by charge exchange.

- A spin filter transmits metastable atoms in different single hyperfine Zeeman states (HFS) corresponding to the magnetic field inside.

- A Lyman- $\alpha$ detector measures the relative HFS occupation numbers after Stark quenching.

The special setup of the horizontally mounted LSP with the vertically injected polarized atomic beam is shown in Fig. 1. In the following, the discussion is mostly limited to the case of hydrogen, whereas some aspects of deuterons are discussed in Sec. VI.

For the ionizer a Glavish-type electron-collision ionizer ${ }^{10}$ was chosen with a magnetic field variable between 0 and $140 \mathrm{mT}$. A sufficiently high field is necessary to decouple electron and nuclear spins in order to conserve the nuclear spin during the ionization process. The atoms from the ABS are ionized by electrons emitted from a hot filament. The electron density is increased by capturing them in a po- 


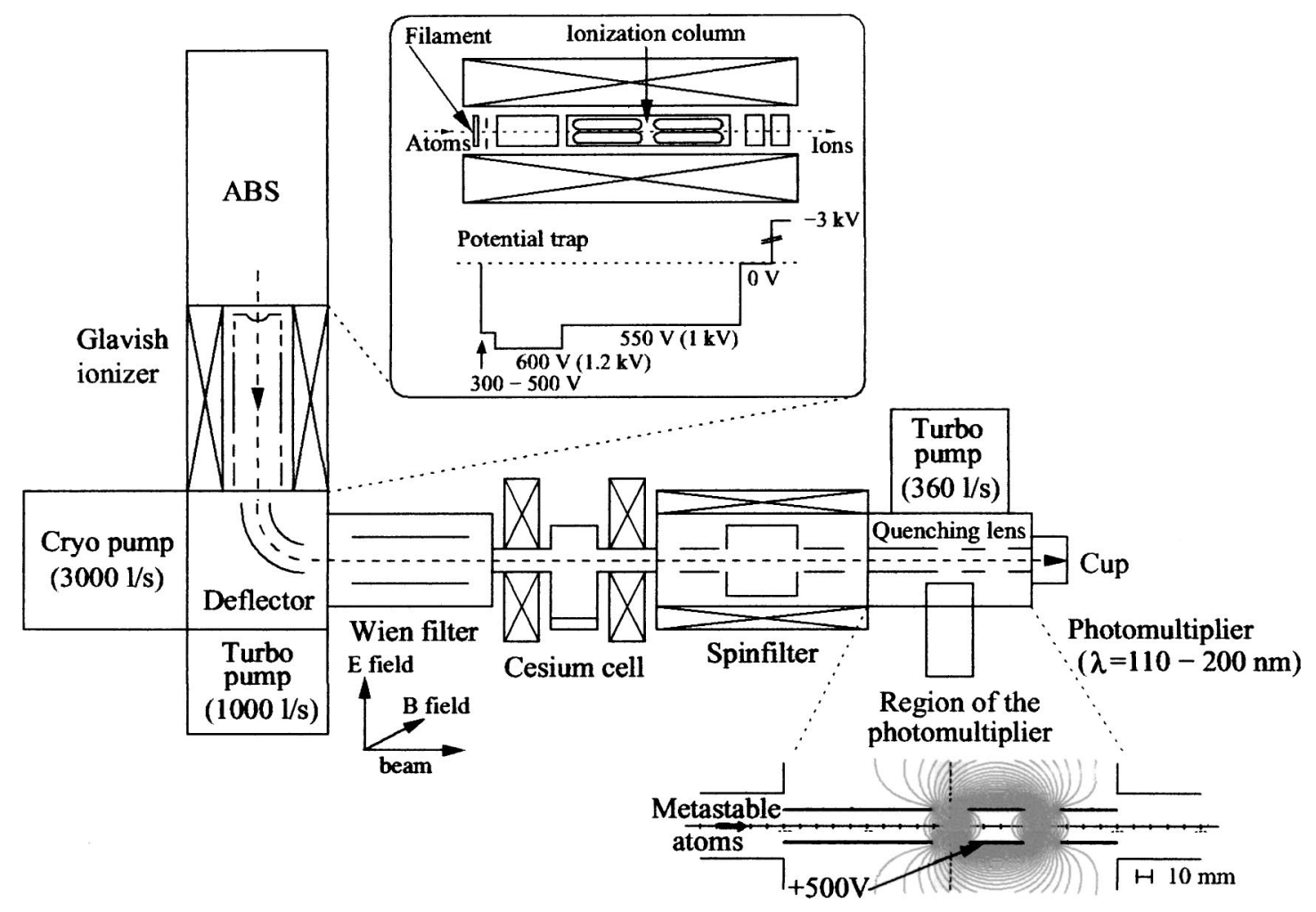

FIG. 1. Setup and principle of the polarization measurement with the Lamb-shift polarimeter. Details show the ionizer and the Stark quenching region.

tential trap produced by several cylindrical electrodes. These are also used to extract and focus the proton (deuteron) beam (see the inset in Fig. 1). Additionally, the gyration of the electrons in the magnetic field increases the ionization efficiency by orders of magnitude to $\sim 10^{-4}$. When potentials between 1 and $2 \mathrm{kV}$ at the ionization column are applied, a plasma can be generated, which leads to a further increase of the ionization efficiency to about $10^{-3}$. The higher potentials reduce also the loss of ions during beam transport. However, this plasma mode is very sensitive to the pressure inside the ionizer.

Subsequently, the ions are electrostatically deflected onto the horizontal beam axis. The necessary $90^{\circ}$ rotation of the polarization direction is accomplished by a Wien filter. Its crossed magnetic and electric fields $B$ and $E$ are produced by coils with iron pole pieces and electric field plates. The spin precession determines $B$, whereas the $E$ field is adjusted to compensate for the beam deflection. Employing electrical lenses in front of and behind the Wien filter, a proton beam transmission of $80 \%$ for a spin precession of $90^{\circ}$ was achieved.

The Wien filter is used as a mass filter as well, which proved to be important for the following reasons:

(1) Only protons can reach the quenching region. Other ions will thus not produce background light near the photomultiplier.

(2) Although the cross section for the process $\mathrm{H}_{2}^{+}+\mathrm{Cs}$ $\rightarrow \mathrm{H}_{(2 S)}+\cdots$ was measured by us to be $70 \pm 10$ times smaller than that for the production of metastable atoms from protons, the remaining contribution of the $\mathrm{H}_{2}^{+}$ions to the background is removed by the Wien filter.
(3) Furthermore, it is very useful for diagnostics, as described in Sec. IV.

By charge exchange with cesium vapor, metastable atoms in the $2 S$ state are produced. ${ }^{11}$ The optimum temperature of the liquid cesium for the production of metastable atoms in our case is $160^{\circ} \mathrm{C}$. A $10 \mathrm{~g}$ charge of cesium lasts several months.

To define the polarization of the metastable hydrogen, a strong magnetic field in the Cs cell is required, i.e., a field strong with respect to the critical field of $B_{c}=6.34 \mathrm{mT}$ for the metastable atoms. ${ }^{12}$ The critical field $B_{c}$ is related to the hyperfine splitting $\Delta W$ of an atomic eigenstate by $B_{C}$ $=\Delta W / 2 \mu_{B}$, where $\mu_{B}$ is the Bohr magneton.

The spin filter ${ }^{13}$ transmits only metastable atoms in single HFSs, depending on the magnetic field. The other metastable atoms are quenched into the ground state by the three-level spin filter interaction, ${ }^{14}$ induced by the combined action of three fields:

(1) A longitudinal magnetic field of a magnitude of either 53.5 or $60.5 \mathrm{mT}$, such that the metastable substates $\beta-$ and $\beta+$ and the short-lived substates $e+$ and $e-$ of the $2 P$ state become degenerate. ${ }^{15}$ For these magnetic fields the transmission of the metastable HFS $\alpha+$ and $\alpha-$ reaches a maximum. Along a distance of $\sim 30 \mathrm{~cm}$, the magnetic fields should be as homogeneous as possible. The measured variation of the magnetic field is about $\pm 0.03 \mathrm{mT}$.

(2) A transverse electric field of about $10 \mathrm{~V} / \mathrm{cm}$ to couple the $2 S$ and $2 P$ substates depending on the energy difference between them (Stark effect). 


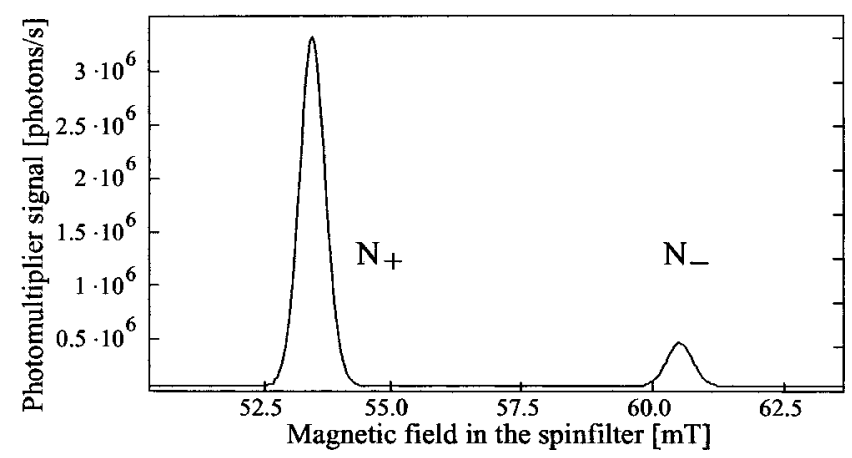

FIG. 2. Lyman- $\alpha$ spectrum of a polarized atomic hydrogen beam of 3 $\times 10^{16}$ atoms/s measured in $20 \mathrm{~s}$.

(3) A radio frequency field of $1.6098 \mathrm{GHz}$ in a $\mathrm{TM}_{010}$ mode cavity for a strong coupling of the metastable substates $\alpha+$ and $\beta+$ at $53.5 \mathrm{mT}$, or $\alpha-$ and $\beta-$ at $60.5 \mathrm{mT}$. The power for this rf field is about $100 \mathrm{~mW}$ and the quality factor $\left(f_{\text {res }} / \Delta f_{\text {FWHM }}\right)$ of our cavity is $\sim 1800$.

Behind the spin filter, the longitudinal electric field $(\sim 100 \mathrm{~V} / \mathrm{cm})$, produced by a cylinder lens, quenches the residual metastable atoms into the ground state. As a function of the magnetic field in the spin filter, the Lyman- $\alpha$ photons $(121 \mathrm{~nm})$, emitted by this transition, are registered selectively with a 1 in. photomultiplier tube (PMT) sensitive only to wavelengths between 110 and $200 \mathrm{~nm}$ (Fig. 2). ${ }^{16}$ The number of photons counted in one hyperfine state is proportional to the number of protons with the same nuclear spin orientation behind the ionizer and, therefore, to the number of atoms in the primary beam.

From the ratio of the number of photons, which are produced in the peaks at 53.5 and at $60.5 \mathrm{mT}$ in the spin filter, the polarization of an atomic or a slow ion beam of 500$2000 \mathrm{eV}$ can be determined. For $3 \times 10^{16}$ atoms/s injected into the ionizer, a peak value of about $3.2 \times 10^{6}$ photons $/ \mathrm{s}$ was registered, thus yielding an overall efficiency of the polarimeter of $\sim 10^{-10}$. A statistical error of the polarization of $\sim 0.005$ is obtained in $2 \mathrm{~s}$.

\section{BACKGROUND IN THE LYMAN SPECTRUM}

For the measurement of the polarization it is essential to minimize the constant background in the Lyman spectrum. This background, produced independently of the magnetic field in the spin filter, stems from several sources:

(1) When ions are not neutralized in the cesium cell, they can reach the quenching region even through the electric fields of the spin filter. These ions are deflected by the electric quenching field and hit an electrode. There they can recombine and, during this process, many photons of different wavelengths are produced in front of the photomultiplier. For example, there are about $600(700)$ possibilities for $\mathrm{O}^{+}\left(\mathrm{N}^{+}\right)$ ions to emit photons in the wavelength window of the photomultiplier. ${ }^{17}$ The Wien filter can be adjusted in a way that only protons (deuterons) arrive at the quenching region. The cylindrical shape of the quenching lens is very helpful, because residual protons do not hit the electrodes, where they would produce photons in front of the photomultiplier.
(2) All ground-state hydrogen atoms in the beam behind the spin filter possess a kinetic energy depending on the potential of the ionization column in the ionizer. Once they hit a surface, they emit photons. It proved necessary to stop atomic and ion beams far away from the quenching region. Therefore, the Faraday cup is placed outside the quenching region. In order to suppress multiply scattered photons, the cup and the quenching lens are made from stainless steel, which has a very small reflection coefficient for wavelengths below $200 \mathrm{~nm}$.

(3) In the spin filter, most of the metastable atoms are quenched into the ground state and Lyman- $\alpha$ photons are produced. A diaphragm between the spin filter and quenching region could be used to prevent this light from reaching the photomultiplier. This aperture, however, would also restrict the diameter of the beam of metastable atoms. The quenching lens prevents a direct line of sight between the spin filter cavity and the entrance window of the photomultiplier, while the choice of the material (stainless steel) suppresses multiple photon scattering.

(4) The background in Fig. 2 is dominated by collisions between beam atoms and the residual gas in the quenching region. The initial signal-to-background ratio is $\sim 100$, it decreases when the chamber pressure is increased. A pressure of $10^{-6} \mathrm{mbar}$ and a beam intensity of $6 \times 10^{12}$ atoms $/ \mathrm{s}$, corresponding to $1 \mu \mathrm{A}$ behind the ionizer, result in 6 $\times 10^{7}$ interactions/s in front of the PMT. With the estimate that $\sim 10 \%$ of the atoms or gas particles produce $110-200$ $\mathrm{nm}$ photons and that $\sim 10^{-3}$ of them are registered in the PMT, about $10^{4}$ background photons/s are expected, in rough agreement with the measured value. The only disadvantage of the cylindrical quenching lens is that it reduces the pumping speed near the quenching volume at the photomultiplier.

(5) A minor source of light is the hot filament in the ionizer. When no ions are produced in the ionizer, a very small signal of about 100 photons/s, varying with the filament temperature, is observed.

When the ionizer operates in the plasma mode, some of the additional photons produced are detected with the photomultiplier. However, the number of photons from this effect is $10^{-4}$ times the number of the expected Lyman- $\alpha$ photons and can normally be neglected.

(6) During all measurements the dark current of the photomultiplier corresponded to $10-100$ photons reaching the PMT within $1 \mathrm{~s}$.

\section{CORRECTIONS}

$$
\begin{aligned}
& \text { The quantity } \\
& P_{\text {Ly }}=\frac{N_{+}-N_{-}}{N_{+}+N_{-}},
\end{aligned}
$$

determined from the peak integrals $N_{+}$and $N_{-}$, is a measure of the nuclear polarization $p_{z}$ of the atoms or ions (Fig. 2). However, a number of corrections are necessary, which are small for ion beams but essential for atomic beams. The precision to which the correction factors can be evaluated determines the absolute accuracy of the polarization value that can be obtained with the LSP. 


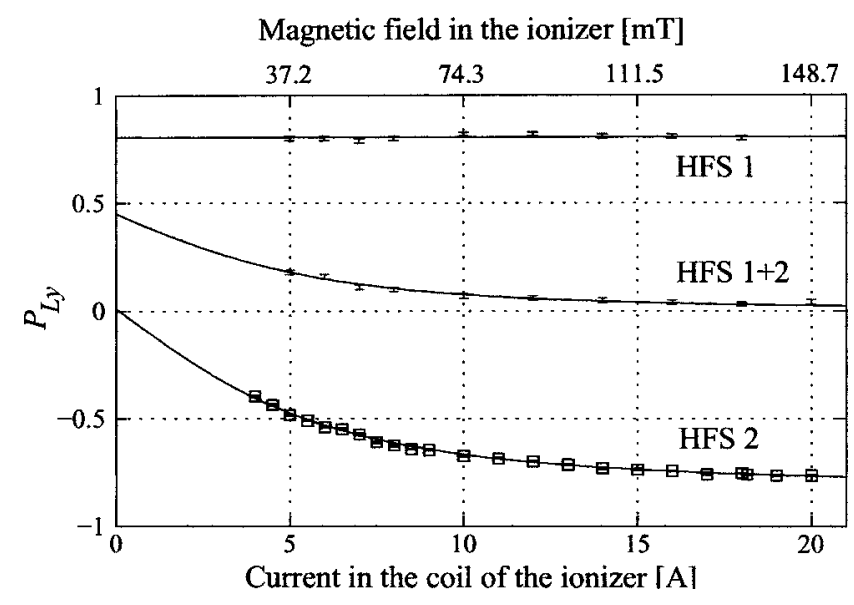

FIG. 3. $P_{\text {Ly }}$ of an atomic hydrogen beam in the HFS 1,2 , and $1+2$ as a function of the current in the solenoid coil of the ionizer.

\section{A. Magnetic field in the ionizer}

While the polarization is constant for hydrogen atoms in the pure HFS 1 and 3, it depends on the magnetic field $B$ for HFS 2 and 4. The ionization process in our ionizer takes place at a magnetic field of $140 \mathrm{mT}$ or less. Therefore, for the calculation of the polarization in arbitrary magnetic fields it is necessary to know the occupation numbers of the atoms in these substates for $B \rightarrow \infty$. In order to derive the occupation numbers of the HFS 2 and 4, the measured value of $P_{\text {Ly }}$ has to be corrected. For a given magnetic field $B$, this correction factor corresponds to

$$
C_{\text {ion }}(B)=\frac{1}{a(B)}=\frac{\sqrt{1+\left(\frac{B}{B_{c}}\right)^{2}}}{\frac{B}{B_{c}}},
$$

where $B_{c}=50.7 \mathrm{mT}$ is the critical field for hydrogen. ${ }^{12}$

The present type of ionizer allows the variation of the magnetic field in the ionization column, whereby the efficiency of the ionizer is affected and the polarization remains the same. Therefore, one can make use of the field dependence of $P_{\text {Ly }}$ of an atomic beam to identify the substates. Even the relative population numbers of a beam composed of a field-independent (pure) and a field-dependent (mixed) substates can be determined, as shown in Fig. 3. For a typical applied magnetic field of $134 \mathrm{mT}$, the correction factor for the HFS 2 is $C_{\text {ion }}=1.070 \pm 0.001$.

\section{B. Residual gas in the ionizer}

The ionizer produces protons from residual gas molecules like $\mathrm{H}_{2}, \mathrm{C}_{\mathrm{n}} \mathrm{H}_{\mathrm{m}}$, and especially from $\mathrm{H}_{2} \mathrm{O}$. These unpolarized protons cannot be separated from the polarized protons produced by the beam and will decrease $P_{\text {Ly }}$. When the atomic beam is switched off, the beam-independent background in the Lyman- $\alpha$ spectrum can be measured separately (Fig. 4). The efficiency of the ionizer has to be the same for both measurements. This condition is fulfilled in the electron impact mode of the ionizer, when the potential of the ionization column is relatively low $(\approx 500 \mathrm{~V})$, resulting in a modest but stable ionization efficiency of about $10^{-4}$. Despite its

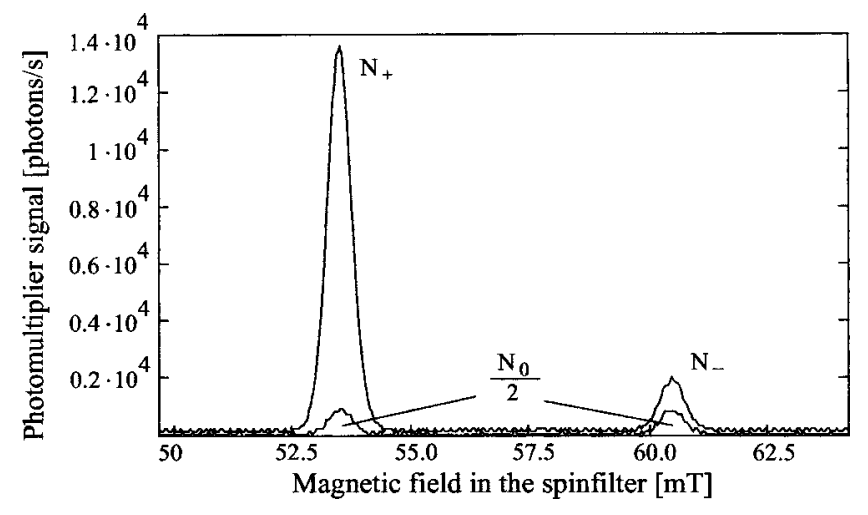

FIG. 4. Lyman- $\alpha$ spectrum with $\left(N_{+}, N_{-}\right)$and without $\left(N_{0}\right)$ a polarized atomic hydrogen beam. The spectrum was obtained with a reduced efficiency of the ionizer (see Sec. V).

higher ionization efficiency, the plasma mode proves not useful here. The plasma's sensitivity to changes in ionizer pressure leads to instabilities, such as changes in ionization efficiency, e.g., when the beam is switched on and off.

With $b=N_{0} /\left(N_{+}+N_{-}\right)$, the ratio of the sums of the peak intensities without and with the beam, the appropriate correction factor can be calculated. The polarization $P$ corrected for this effect is

$$
\begin{aligned}
P & =\frac{\left(N_{+}-N_{0} / 2\right)-\left(N_{-}-N_{0} / 2\right)}{\left(N_{+}-N_{0} / 2\right)+\left(N_{-}-N_{0} / 2\right)} \\
& =\frac{N_{+}-N_{-}}{\left(N_{+}+N_{-}\right)-N_{0}} \\
& =\frac{N_{+}-N_{-}}{\left(N_{+}+N_{-}\right)(1-b)}=P_{\text {Ly }} \frac{1}{1-b} .
\end{aligned}
$$

Thus, the residual gas correction factor is

$$
C_{\text {gas }}=\frac{1}{1-b} \text {. }
$$

Figure 4 shows a spectrum taken after a few hours of pumping only, yielding $C_{\text {gas }}=1.120 \pm 0.002$. Due to the dependence on the vacuum conditions, this factor must be checked repeatedly. After a few days, the pressure in the ionizer without the beam is normally about $2 \times 10^{-8} \mathrm{mbar}$ and the correction factor, typically, drops to $C_{\mathrm{gas}} \approx 1.005$.

\section{Recombination}

When the atoms hit a surface in the ionizer, they can recombine into molecules. From the measured time constant for equilibrium of the ionizer pressure of about $1 \mathrm{~s}$ (after the injection of atomic beam), the number of wall collisions of molecules in the ionizer is estimated to be at least 10000 . Using the field dependence of the depolarization of hydrogen molecules from Ref. 18, an upper limit for the nuclear polarization of the hydrogen molecules of $\sim 0.2 \%$ is calculated. Taking, in addition, into account the known higher depolarization of hydrogen atoms on the stainless steel surface of the ionizer prior to recombination, ${ }^{19}$ we conclude that the nuclear polarization of the molecules in the ionizer is zero. The ionizer will, therefore, produce unpolarized protons and 


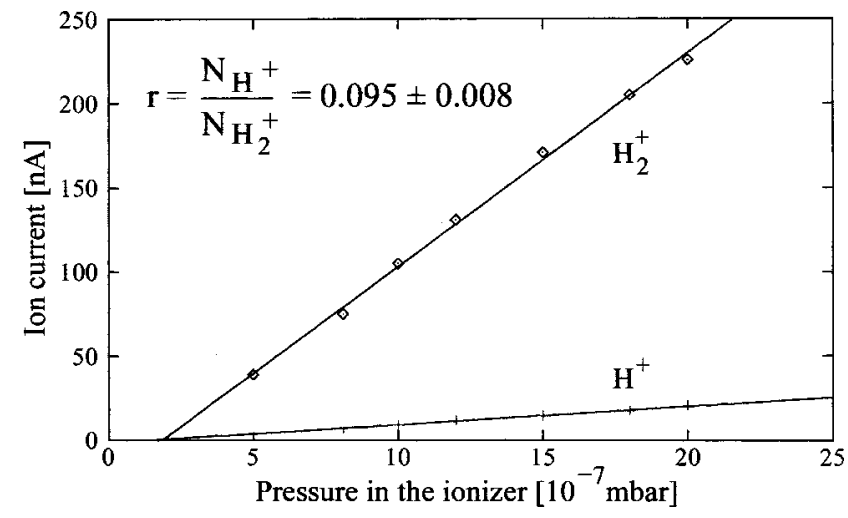

FIG. 5. $\mathrm{H}_{2}^{+}$and the $\mathrm{H}^{+}$ion current as a function of the pressure in the ionizer by admission of varying amounts of $\mathrm{H}_{2}$ into the ionizer.

$\mathrm{H}_{2}^{+}$ions from these molecules. Using the Wien filter, it is possible to determine this beam-dependent unpolarized background. A calibration of the ionization process is obtained by measuring the proton and $\mathrm{H}_{2}^{+}$ion currents as a function of the ionizer pressure, which is varied by admission of a small amount of hydrogen gas (Fig. 5). These currents are compared with those from an atomic beam in the ionizer. Since the ratio $r$ of the number of protons and $\mathrm{H}_{2}^{+}$ions now is known, it is straightforward to measure the $\mathrm{H}_{2}^{+}$current with the atomic beam on and then to determine the current of unpolarized protons in the ion beam, produced from $\mathrm{H}_{2}$, with use of Fig. 5. In our case, the ratio of the number of protons from molecules to that of all protons is

$$
c=\frac{N_{\text {protons from } \mathrm{H}_{2}}}{N_{\text {protons }}}=\frac{N_{\mathrm{H}_{2}^{+}} r}{N_{\text {protons }}}=0.087 \pm 0.007 .
$$

The correction factor arising from this effect is

$$
C_{\text {rec }}=\frac{1}{1-c}=1.095 \pm 0.008 .
$$

When the atomic beam enters the ionizer, the pressure inside increases from $2 \times 10^{-8}$ to $2 \times 10^{-6}$ mbar.

Using the fact that both cross sections have the same energy dependence in the region of interest and differ only in magnitude by a factor of 0.6 , it is possible to calculate the efficiency of the ionizer from the data of Fig. $5 .^{20}$

\section{Wien filter}

The precession of the polarization of the protons depends on the quantity $\int B d l$ along their trajectories in the magnetic field of the Wien filter (WF). This field integral varies according to differences in the path lengths and field inhomogeneities. This leads to some loss of polarization due to the averaging over different spin directions. In first order, the loss increases linearly with the strength of the magnetic field, i.e., with the precession angle. A comparison of the minima and maxima of the curve in Fig. 6 yields a polarization loss of $2 d=0.016 \pm 0.004$ for a precession of the polarization from $\beta=+90^{\circ}$ to $+270^{\circ}$. For a precession from $\beta$ $=0^{\circ}$ to $90^{\circ}$ at $I=1.55 \mathrm{~A}$, the correction factor amounts to

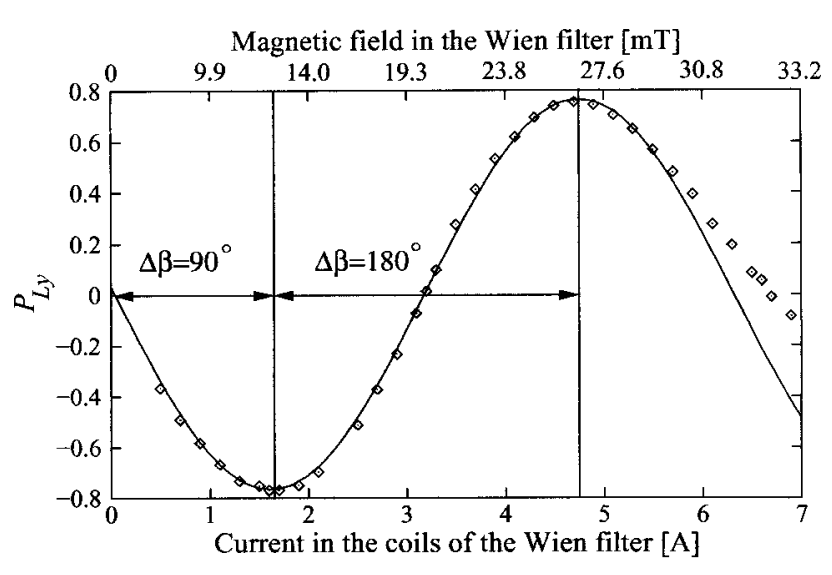

FIG. 6. Measured $P_{\text {Ly }}$ as a function of the current in the coils of the Wien filter.

$$
C_{\mathrm{WF}}=1+\frac{d}{P_{\mathrm{Ly}}}=1.010 \pm 0.003 .
$$

\section{E. Cesium cell}

When protons with polarization $P$ pick up unpolarized electrons from the cesium atoms in the vapor cell, the occupation numbers of metastable atoms in the mixed HFS $\alpha-$ and $\beta+$ depend on the magnetic field $B$ in the cell, and therefore $P_{\text {Ly }}$ as well.

With the probabilities $W_{+}=(1+P) / 2$ and $W_{-}=(1$ $-P) / 2$ to find a proton with $m_{I}=+1 / 2$ or $m_{I}=-1 / 2$ in the beam the probability $W_{\alpha+}$ for the production of metastable atoms in HFS 1 is

$$
W_{\alpha+}=\epsilon W_{+} W_{m_{J}=+1 / 2}=\epsilon\left(\frac{1+P}{4}\right),
$$

where $\epsilon$ is the efficiency of populating a metastable state, and $W_{m_{J}=+1 / 2}=1 / 2$ is the probability of capturing an electron with $s=+1 / 2$.

For the mixed HFS $\alpha-$, the situation is more complicated because both, $\left(m_{I} ; m_{J}\right)=(+1 / 2 ;-1 / 2)$ or $(-1 / 2$; $+1 / 2$ ) contribute to

$$
\begin{aligned}
W_{\alpha-}(B) & =\epsilon\left[\left(\frac{W_{+}}{2} \frac{1-a(B)}{2}\right)+\left(\frac{W_{-}}{2} \frac{1+a(B)}{2}\right)\right] \\
& =\epsilon\left[\frac{(1+P)[1-a(B)]}{8}+\frac{(1-P)[1+a(B)]}{8}\right],
\end{aligned}
$$

where $a(B)$ is defined in Eq. (1).

The measured $P_{\text {Ly }}$ is given by

$$
\begin{aligned}
P_{\text {Ly }}(B) & =\frac{W_{\alpha+}-W_{\alpha-}}{W_{\alpha+}+W_{\alpha-}} \\
& =\frac{W_{+}[1+a(B)]-W_{-}[1+a(B)]}{W_{+}[3-a(B)]+W_{-}[1+a(B)]} \\
& =\frac{P[1+a(B)]}{2+P[1-a(B)]} .
\end{aligned}
$$

The proton polarization $P$ can be expressed as a function of the known magnetic field $B_{\mathrm{Cs}}$ and the measured $P_{\mathrm{Ly}}$, 


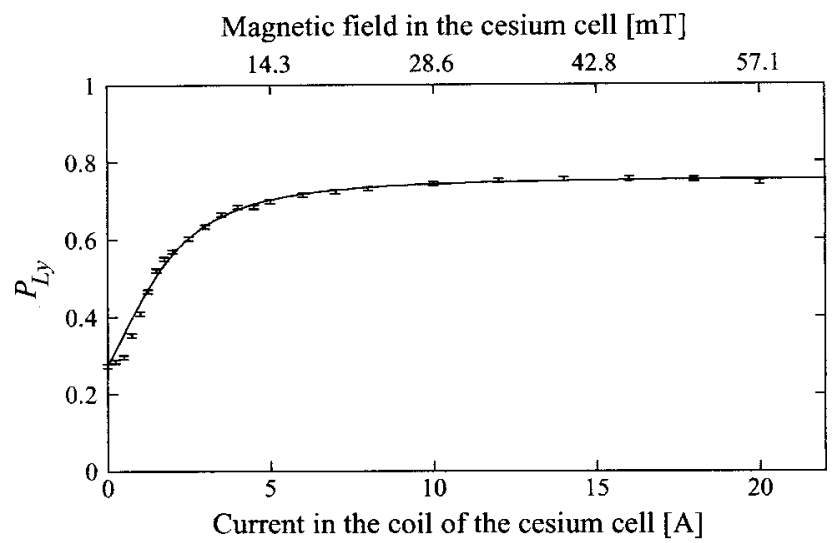

FIG. 7. Measured and fitted $P_{\text {Ly }}$ [Eq. (6)] as a function of the current in the field coils of the cesium cell.

$$
P=\frac{2}{[1+a(B)]-P_{\mathrm{Ly}}[1-a(B)]} P_{\mathrm{Ly}} .
$$

The correction factor $C_{\mathrm{Cs}}$ is thus a function of the magnetic field and of the measured $P_{\mathrm{Ly}}$,

$$
C_{\mathrm{Cs}}=\frac{2}{[1+a(B)]-P_{\mathrm{Ly}}[1-a(B)]} .
$$

In our cesium cell, average magnetic fields up to $B$ $=55 \mathrm{mT}$ can be produced, which correspond to nine times the critical field of the metastable hydrogen $\left(B_{c}\right.$ $=6.34 \mathrm{mT}$ ). Therefore, during the production of the metastable atoms $a=0.9934 \pm 0.0004$ of the polarization is conserved. For $P_{\mathrm{Ly}}=0.78$ the correction factor is $C_{\mathrm{Cs}}=1.0058$ \pm 0.0004 . This can be determined very precisely from the difference between the measured $P_{\text {Ly }}$ and the extrapolated value $P_{\text {Ly }}(B \rightarrow \infty)$ (Fig. 7). The discrepancy between measurement and fit for low currents in the cesium cell coils is caused by the remanent magnetization of the stainless steel of the cesium cell.

\section{F. Spin filter}

Even if measured with an unpolarized primary proton beam or with unpolarized molecular $\mathrm{H}_{2}$ gas in the ionizer, the Lyman spectrum shows some asymmetry. This is caused in the spin filter by differences in the magnetic field inhomogeneities at 53.5 and $60.5 \mathrm{mT}$, leading to different transmission probabilities $t_{\alpha+}$ and $t_{\alpha-}$ for metastable atoms in HFS $\alpha+$ or HFS $\alpha-$, respectively.

The polarization $P_{\text {Meta }}$ of metastable atoms in the cesium cell, corrected for $B_{\mathrm{Cs}} \rightarrow \infty$ is used to calculate the probabilities of metastable atoms in HFS $\alpha+(\alpha-)$ in front of the spin filter,

$$
W_{\alpha+}=\frac{1+P_{\text {Meta }}}{2} \text { and } W_{\alpha-}=\frac{1-P_{\text {Meta }}}{2} .
$$

For the general case including $t_{\alpha+} \neq t_{\alpha-}$,

$$
P_{\mathrm{Ly}}=\frac{t_{\alpha+} W_{\alpha+}-t_{\alpha-} W_{\alpha-}}{t_{\alpha+} W_{\alpha+}+t_{\alpha-} W_{\alpha-}} .
$$

Using $T \equiv t_{\alpha+} / t_{\alpha-}$, one obtains

$$
P_{\mathrm{Ly}}=\frac{T W_{\alpha+}-W_{\alpha-}}{T W_{\alpha+}+W_{\alpha-}}=\frac{P_{\text {Meta }}(T+1)+(T-1)}{P_{\text {Meta }}(T-1)+(T+1)},
$$

from which follows

$$
P_{\text {Meta }}=\frac{P_{\mathrm{Ly}}(1+T)+(1-T)}{P_{\mathrm{Ly}}(1-T)+(1+T)} .
$$

The correction factor $C_{\mathrm{SF}}$ for the spin filter $(\mathrm{SF})$ is a function of $T$ and the measured $P_{\text {Ly }}$,

$$
C_{\mathrm{SF}}=\frac{(1+T)+\frac{1}{P_{\mathrm{Ly}}}(1-T)}{P_{\mathrm{Ly}}(1-T)+(1+T)} .
$$

The transmission ratio $T$ is obtained from a measurement of $P_{\text {Ly }}$ with an unpolarized beam $\left(P_{\text {Meta }}=0\right)$ using Eq. (8).

For our spin filter, $P_{\text {Ly }}\left(P_{\text {Meta }}=0\right)=0.0044 \pm 0.0020$, which yields $T=1.009 \pm 0.003$. For typical values of $P_{\mathrm{Ly}}$ $=0.78$ and -0.78 , one finds $C_{\mathrm{SF}}=0.997 \pm 0.002$ for $\alpha+$, and $1.003 \pm 0.002$ for $\alpha-$. This correction factor is the only one that reduces a positive polarization, or increases a negative polarization.

In Ref. 4 the authors simply normalized the polarized Lyman- $\alpha$ spectrum to the unpolarized one.

\section{G. Other corrections necessary?}

Below we discuss some other effects that could influence $P_{\text {Ly }}$ :

(1) It is known that the cross section for the ionization process

$$
\mathrm{H}+e \rightarrow \mathrm{H}^{+}+2 e,
$$

depends on both the polarization of atoms and electrons. ${ }^{21}$ If electrons are polarized, the ionization efficiency for atoms in different HFS can vary depending on the type of ionizer.

- In Ref. 22 it is reported that in an electron cyclotron resonance (ECR) ionizer the number of protons produced depends on the hyperfine states of the incoming atoms. Some of the accelerated electrons stem from polarized atoms and, therefore, the electrons in the ionization plasma may not be completely unpolarized. Polarization transfer from elastic electron scattering off the polarized atoms may contribute as well. ${ }^{23}$ The ionization efficiency depends on the HFS of the primary atoms. Differences on the order of $0.01 \%-0.1 \%$ have been observed. Thus, it was possible to tune the transition units in the polarized ABS.

- The authors of Ref. 24, using a strong field electronbombardment ionizer, measured changes of the ionization efficiency of $1 \%$ for different combinations of atoms in two hyperfine states.

For the Glavish-type ionizer, the effect described above should be much weaker, because almost all electrons originate from a hot filament and are unpolarized. The influence of polarized electrons in the ionizer can be determined by measuring the ion current in the cup as a function of the occupation numbers of the HFS, when the heating of the cesium cell is switched off. The ion current will change, depending on the rf 
TABLE I. Correction factors for HFS 1 and 2.

\begin{tabular}{lcc}
\hline \hline Correction factor & HFS 1 & HFS 2 \\
\hline Ionizer: $B \neq \infty\left(C_{\text {ion }}\right)$ & 1 & $1.070 \pm 0.001$ \\
Residual gas $\left(C_{\text {gas }}\right)$ & \multicolumn{2}{c}{$1.005 \pm 0.002$} \\
Recombination $\left(C_{\text {rec }}\right)$ & \multicolumn{2}{c}{$1.095 \pm 0.008$} \\
Wien filter $\left(C_{\mathrm{WF}}\right)$ & \multicolumn{2}{c}{$1.008 \pm 0.002$} \\
Cs cell $\left(C_{\mathrm{Cs}}\right)$ & \multicolumn{2}{c}{$1.0054 \pm 0.0004$} \\
Spin filter $\left(C_{\mathrm{SF}}\right)$ & $0.997 \pm 0.002$ & $1.003 \pm 0.002$ \\
Total $C_{\text {tot }}$ & $1.112 \pm 0.010$ & $1.197 \pm 0.010$ \\
\hline \hline
\end{tabular}

transition units employed behind the last sextupole magnet in the ABS, by which the occupation numbers of the HFS can be varied without changing the absolute flux.

(2) When the metastable atoms enter the region of the magnetic field gradient at the exit of the cesium cell, transverse magnetic field components cause a precession of the polarization depending on the atom trajectories. Partly, the small loss of polarization is compensated by the field gradient of opposite direction at the entrance of the spin filter, as calculated for the LSP of the TUNL laboratory. ${ }^{25}$ Our design maintains the same absolute gradients of $\approx 10 \mathrm{mT} / \mathrm{cm}$ at the cesium cell and at the spin filter. The field gradient at the exit of the cesium cell was varied around this value by applying iron shims of different thickness at the end of the cesium cell coil. No measurable change in the Lyman spectra could be observed.

(3) It has been shown ${ }^{11}$ that the distribution of photons from quenching of polarized metastable atoms in a homogeneous electric field is nonisotropic due to the electric dipole character of the ground-state transition. For a Lamb-shift polarimeter this effect is very small, because only metastable atoms in HFS $\alpha+$ and $\alpha$ - are quenched in front of the photomultiplier, which have the same electron-spin orientation for different orientations of the nuclear spin.

\section{H. Calculation of the polarization}

From the known correction factors for atoms in the different HFS it is possible to determine the polarization $p_{z(z)}$ of the atomic beam from the measured $P_{\mathrm{Ly}}$. In principle, it is necessary to calculate the polarization of the metastable atoms in front of the spin filter with the correction factor $C_{\mathrm{SF}}$ and in front of the cesium cell with the correction factor $C_{\mathrm{Cs}}$, because these factors are functions of the polarization. The corrections are, however, very small. It is, therefore, sufficient to multiply $P_{\text {Ly }}($ HFS $)$ by the single HFS-dependent correction factors, listed in Table I.

From the measured quantity $P_{\text {Ly }}=0.780 \pm 0.002$ (Fig. 2) and with the total correction factor, the nuclear polarization of the atomic beam (HFS 1) is calculated to be $p_{z}=0.867$ \pm 0.009 .

For a polarized proton (or deuteron) beam, the correction factors $C_{\text {gas }}, C_{\text {rec }}$, and $C_{\text {ion }}$, associated with the ionization process, are not applicable. In this case, the total correction

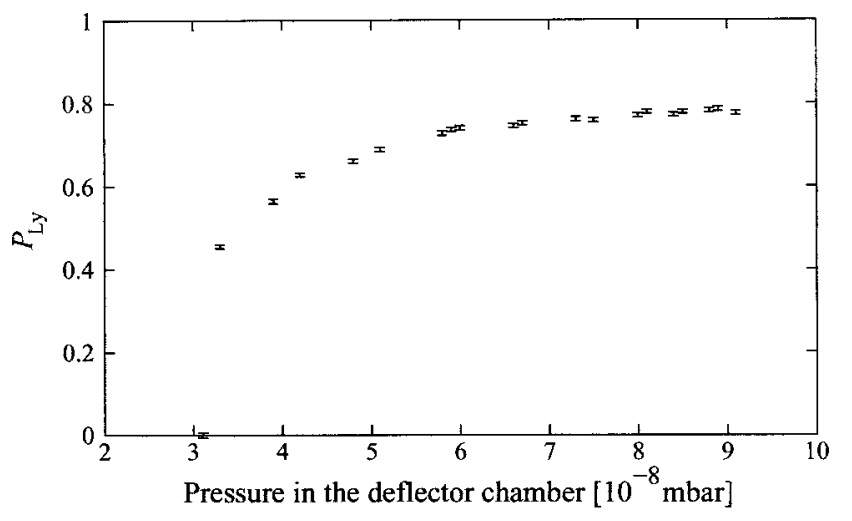

FIG. 8. $P_{\mathrm{Ly}}$ as a function of the atomic beam intensity, measured in units of the absolute pressure in the deflector chamber. Without the beam, the pressure in the deflector chamber was $3.1 \times 10^{-8}$ mbar.

$C_{\mathrm{WF}} C_{\mathrm{Cs}} C_{\mathrm{SF}}=1.011 \pm 0.003$ is much smaller than for the atomic case.

\section{SENSITIVITY}

The measurements, discussed here, profited from the high beam intensity of the ANKE ABS. For hydrogen, typically, $7.4 \times 10^{16}$ atoms/s in two HFS are injected into a compression tube of $10 \mathrm{~mm}$ diam and $100 \mathrm{~mm}$ length. The LSP is, however, sensitive enough to measure the polarization of a beam with strongly reduced intensity in a reasonable time. As an example, Fig. 4 shows a spectrum, measured with full beam intensity, but with the ionizer efficiency reduced from $10^{-3}$ to $10^{-5}$, resulting in a photon rate of $10^{4}$ photons/s at $53.5 \mathrm{mT}$. With decreasing beam intensity the contribution of unpolarized atoms from residual gas to the Lyman spectrum increases. The measured asymmetry $P_{\text {Ly }}$ thus decreases, as shown in Fig. 8. As discussed in Sec. IV A, the nuclear beam polarization can be determined by applying the proper corrections even for the low beam intensity. These corrections do not appear in polarization measurements on proton (or deuteron) beams and should give good results for intensities as low as $10^{11}$ ions/s (20 nA). Possible improvements are discussed in the next section.

\section{DISCUSSION}

With the Lamb-shift polarimeter, described here, it is possible to measure the polarization of a hydrogen beam with an error of $1 \%$ in $20 \mathrm{~s}$ or less, depending on the atomic beam intensity. The statistical error of about $0.3 \%$ increases to $0.5 \%$ when the time period for one scan is reduced to $2 \mathrm{~s}$.

The total error of the nuclear polarization is dominated by the systematic error of about $0.9 \%$ in the correction factors for the present LSP. As discussed above, the corrections have to take into account the properties of the LSP components, but they can be determined using the polarimeter itself. The largest contribution to the systematic error of $0.8 \%$ originates from the recombination of beam atoms in the ionizer. This error can be reduced by providing a better vacuum in the ionizer. 


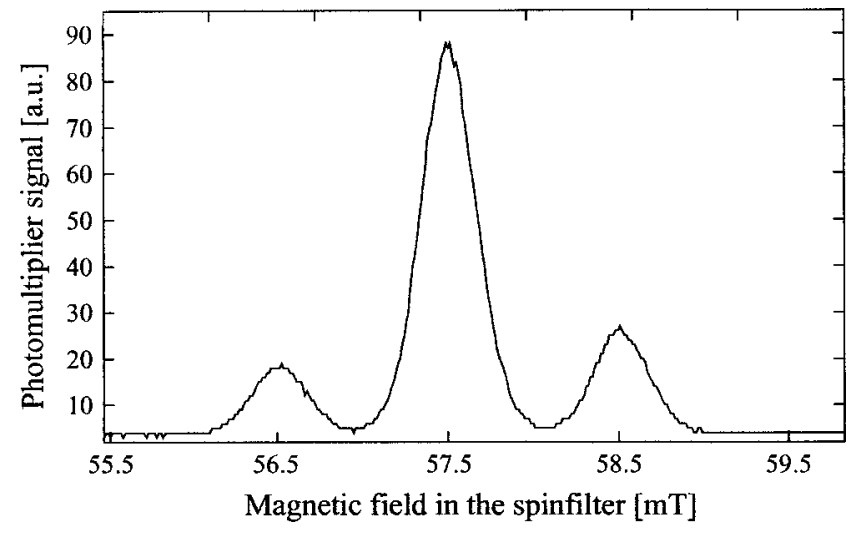

FIG. 9. Lyman- $\alpha$ spectrum of a polarized deuterium beam. $\left(P_{z \mathrm{Ly}}\right.$ $\left.=-0.06 ; P_{z z \mathrm{Ly}}=-1.09\right)$.

The total error of the polarization of a proton (deuteron) beam of $500 \mathrm{nA}$ is $\Delta p_{z(z)}=0.004$ for a $2 \mathrm{~s}$ measurement period.

It is essential to minimize the fraction of molecules in the atomic beam, because unpolarized nucleons reduce the polarization of the gas target. With the present ionizer, it was not possible to separate $\mathrm{H}_{2}$ molecules in the beam from those produced by recombination in the ionizer. Their density is estimated to be higher by two orders of magnitude than that of $\mathrm{H}_{2}$ molecules injected directly with the beam from the ABS. With additional pumping of the ionization volume, this molecular background from recombination in the ionization column should be substantially reduced. It will then be possible to measure the molecular fraction in the beam with a chopper in the beam line and the Wien filter of the LSP.

For measurements of the vector and tensor polarization of a deuterium beam, the LSP is expected to enable a precision comparable to that obtained for hydrogen. Some of the correction factors are lower for deuterium than for hydrogen. Because of the smaller critical magnetic field (11.4 mT compared to $50.7 \mathrm{mT}$ for hydrogen), the polarization is conserved to a higher degree during ionization as well as during charge exchange. The separation of the individual HFS peaks in the Lyman spectrum as a function of the magnetic field in the spin filter is about $1 \mathrm{mT}$ only (Fig. 9). Therefore, the spin filter correction, caused by different HFS transmissions due to magnetic field inhomogeneities, becomes negligible. In addition, the background in the Lyman spectra cannot be affected by deuterium atoms produced from the residual gas, e.g., $\mathrm{H}_{2} \mathrm{O}$.

The present polarimeter setup was crucial for the development and fine tuning of the rf-transition units of the ANKE ABS. For deuterium beams, it is expected that the LSP will be much more important. In particular, the capability of fast online polarization monitoring in connection with the excellent signal-to-background ratio, proved valuable.

Further improvements are scheduled to increase the total polarimeter sensitivity. In order to reduce the unwanted background, a new ionizer with a nonevaporable getter pump of 2000 1/s (Ref. 26), around the ionization volume has been built. It also provides a stronger magnetic field of up to 200 $\mathrm{mT}$. We expect to achieve a reduction of the total error to $0.7 \%$ in a measurement of $2 \mathrm{~s}$ only. A shorter quenching region and a new Wien filter are under construction. They will lead to a better beam transport of the metastable atoms and higher transmission, respectively. In addition, the present photomultiplier of 1 in. diameter is replaced by a 2 in. type with a narrower window of sensitivity around the $121 \mathrm{~nm}$ Lyman- $\alpha$ wavelength. ${ }^{16}$ Moreover, a lock-in amplifier in combination with a chopper in front of the ionizer will separate the beam-associated Lyman- $\alpha$ signal from the background. With these improvements, it appears feasible to measure continuously the polarization of a small fraction of the atoms, emerging from the storage-cell target of the ANKE spectrometer into the LSP, in order to determine the polarization of the hydrogen and deuterium targets. The LSP constitutes an indispensable tool for future polarization experiments, such as the measurement of the tensor analyzing power of the proton-induced deuteron-breakup reaction ${ }^{27}$ at COSY, but also for other polarized-ion source developments.

\section{ACKNOWLEDGMENTS}

The authors wish to acknowledge the financial support by the German ministry BMBF (06 OK $862 \mathrm{I}$ and $06 \mathrm{OK}$ 958) and FZ Jülich (FF\&E Contract No. 41445283). The authors thank W. Haeberli for the loan of the deflector, TRIUMF and LANL for the spin filter solenoid and the cavity, and T. B. Clegg for valuable comments on the manuscript of this article.

${ }^{1}$ Yu. A. Pliss and L. M. Soroko, Nucl. Instrum. Methods 135, 497 (1976).

${ }^{2}$ A. N. Zelenskii, S. A. Kokhanovskii, V. M. Lobashev, and V. G. Polushkin, Nucl. Instrum. Methods Phys. Res. A 245, 223 (1986).

${ }^{3}$ A. S. Belov, S. K. Yesin, S. A. Kubalov, V. E. Kuzik, A. A. Stepanov, and Y. P. Yakushev, Nucl. Instrum. Methods Phys. Res. A 255, 442 (1987).

${ }^{4}$ A. J. Mendez, C. D. Roper, J. D. Dunham, and T. B. Clegg, Rev. Sci. Instrum. 67, 3073 (1996).

${ }^{5}$ P. D. Eversheim, M. Altmeier, O. Felden, M. Glende, and R. Gebel, in Proceedings of the 8th International Workshop on Polarized Sources and Targets (PST99), Erlangen, edited by A. Gute, S. Lorenz, and E. Steffens (Universität Erlangen-Nürnberg, 2000), p. 142.

${ }^{6}$ I. Nishikawa et al., Proceedings of the RCNP Workshop on Spin Polarized Nuclear Fusion, POLUSION99, RNCP, edited by M. Tanaka, Osaka University, 1999, p. 81.

${ }^{7}$ H. R. Kremers, F. Barzangy, R. Bieber, and A. G. Drentje, in Proceedings of the 8th International Workshop on Polarized Sources and Targets (PST99), Erlangen, edited by A. Gute, S. Lorenz, and E. Steffens (Universität Erlangen-Nürnberg, 2000), p. 82.

${ }^{8}$ M. Mikirtytchiants et al., in Proceedings of the 9th International Workshop on Polarized Sources and Targets (PSTO1), Nashville, edited by V. P. Derenchuk and B. Przewoski (World Scientific, Singapore, 2002), p. 47.

${ }^{9}$ C. Baumgarten et al., Nucl. Instrum. Methods Phys. Res. A 482, 606 (2002).

${ }^{10}$ H. F. Glavish, Nucl. Instrum. Methods 65, 1 (1968).

${ }^{11}$ P. Pradel, F. Roussel, A. S. Schlachter, G. Spiess, and A. Valance, Phys. Rev. A 10, 797 (1974).

${ }^{12}$ W. Haeberli, Annu. Rev. Nucl. Sci. 17, 373 (1967).

${ }^{13}$ J. L. McKibben, G. P. Lawrence, and G. G. Ohlsen, Phys. Rev. Lett. 20, 1180 (1968)

${ }^{14}$ J. L. McKibben, Am. J. Phys. 45, 1022 (1977).

${ }^{15} \mathrm{Here}$, and in later sections, the following notation is used for the hydrogen HFS:

$$
\begin{aligned}
& \left.\left.\alpha+\text { for } 2 S_{1 / 2} \mid m_{J}=+\frac{1}{2}, m_{I}=+\frac{1}{2}\right)\right\rangle \text { and in shorter form } \\
& \alpha-\text { for } 2 S_{1 / 2}\left|+\frac{1}{2},-\frac{1}{2}\right\rangle, \quad \beta-\text { for } 2 S_{1 / 2}\left|-\frac{1}{2},-\frac{1}{2}\right\rangle, \\
& \beta+\text { for } 2 S_{1 / 2}\left|-\frac{1}{2},+\frac{1}{2}\right\rangle, \mathbf{e}+\text { for } 2 P_{1 / 2}\left|+\frac{1}{2},+\frac{1}{2}\right\rangle, \\
& \mathbf{e}-\text { for } 2 P_{1 / 2}\left|+\frac{1}{2},-\frac{1}{2}\right\rangle, \quad \mathbf{1} \text { for } 1 S_{1 / 2}\left|+\frac{1}{2},+\frac{1}{2}\right\rangle, \\
& \mathbf{2} \text { for } 1 S_{1 / 2}\left|+\frac{1}{2},-\frac{1}{2}\right\rangle, \mathbf{3} \text { for } 1 S_{1 / 2}\left|-\frac{1}{2},-\frac{1}{2}\right\rangle, \text { and }
\end{aligned}
$$


4 for $1 S_{1 / 2}\left|-\frac{1}{2},+\frac{1}{2}\right\rangle$.

${ }^{16}$ Electron Tubes, Model Nos. 9424B (2 in.) and 9403 (1 in.), available from Electron Tubes Lim., Bury Street, Ruislip Middx HA4 7TA, U.K.

${ }^{17}$ National Institute of Standards and Technology (2002); http:// physics.nist.gov/cgi-bin/AtData/main_asd

${ }^{18}$ T. Wise et al., Phys. Rev. Lett. 87, 042701 (2001).

${ }^{19}$ J. S. Price and W. Haeberli, Nucl. Instrum. Methods Phys. Res. A 349, 321 (1994).

${ }^{20}$ National Institute of Standards and Technology (2002); electron-impact cross section data base; http://physics.nist.gov/PhysREfData/Ionization

${ }^{21}$ M. J. Alguard, V. W. Hughes, M. S. Lubell, and P. F. Wainwright, Phys. Rev. Lett. 39, 334 (1977).

${ }^{22}$ E. R. Crosson, T. B. Clegg, H. J. Karwowski, and S. K. Lemieux, Nucl. Instrum. Methods Phys. Res. A 310, 703 (1991).

${ }^{23}$ P. G. Burke and J. F. B. Mitchell, J. Phys. B 7, 214 (1974).

${ }^{24}$ S. Jaccard, AIP Conf. Proc. 80, 95 (1982).

${ }^{25}$ W. J. Thompson, Nucl. Instrum. Methods Phys. Res. A 333, 443 (1993).

${ }^{26}$ SAES Getters, 20020 Lainate, Milano, Italy.

${ }^{27}$ V. Komarov et al., Phys. Lett. B 553, 179 (2003). 